This version is the one submitted for review, it is not the final version for publication in the journal of FCAA.

\title{
PLC-BASED DISCRETE FRACTIONAL-ORDER CONTROL DESIGN FOR AN INDUSTRIAL-ORIENTED WATER TANK VOLUME SYSTEM WITH INPUT DELAY
}

\author{
Arkadiusz Mystkowski ${ }^{1}$, Argyrios Zolotas ${ }^{2}$
}

\begin{abstract}
We present PLC-based fractional-order controller design for an industrial -oriented water tank volume control application. The system comprises input delay which is a typified characteristic in such industrial process control applications. The particular contribution of this work is on discrete fractional-order PID implementation via PLC and its application to the aforementioned realistic water tank test bed. Stability and robustness properties of fractional-order discrete PID feedback-loops for different approximation methods and orders are also shown. Fractional-order controllers are obtained for a variety of stability margin choices, and benefits of the non-integer-order controllers compared to the integer-order PID control are illustrated via simulation and experimental runs on a realistic test-bed.

MSC 2010: Primary 26A33; Secondary 93C55, 34A08, 93B52, 93C35, 93C95

Key Words and Phrases: fractional-order control; PID control; PLC; water volume control; input delayed systems; MPS Compact Workstation.
\end{abstract}

\section{Introduction}

Control practitioners, when it comes to designing simple controller structures for the relevant control applications as is the case in process control industry, face challenges in understanding the process to control in conjunction to design and operational experience (a common practice for many years in tuning simple PI/PD/PID feedback loops in the aforementioned industry). Practices tend to change nowadays given the advances in software tools, simulation packages and the deeper understanding of design

(C) Year Diogenes Co., Sofia

pp. $\mathrm{xxx}-\mathrm{xxx}, \mathrm{DOI}$ : 
methodologies. Obtaining mathematical models is now a common practice and it can be based both on physical laws and mathematical equations or via identification from experimental data. In fact, designs advance a step further to include more elegant fractional-order models and associated analysis and design tools for both linear and nonlinear control systems [1], [15].

Hence, fractional calculus (in particular via fractional-order control methods) becomes even more popular in terms of addressing modeling, analysis and design of systems. Fractional-order methods have a long standing theoretical foundation, i.e. commencing with fractional calculus in 1695. The foundation on fractional calculus started with Liouville, Holmgren and Riemann, Grunwald, Letnikov, Riemann and others see for example [4, 16]. A number of problem investigations started soon after, i.e. Abel in 1823 investigated the solution of the integral equation for the tautochrone, which is more economical and useful than the conventional integer order approach [16]. The problem of synthesis and analysis of control systems described by fractional-order differential or difference equations are considered in the last couple of decades, i.e. examples can be seen in [1], [10], [12], 15], [17], [18, also in terms of tuning [5] and a useful survey [6]. In particular, Lanusse et al [19] discussed on a PLC implementation of a robust CRONE controller for a Festo Compact Workstation hydraulic pressure control system. Their CRONE controller was implemented in the S7-300 Simatic, Siemens controller, using the ladder-diagram language for the controller algorithm. The nature of the paper presented here is different to that of [19] and hence forms novel contribution to the topic, both from the type of controller and its PLC implementation (language used) viewpoint as well as the way the discetisation process and its impact to the robustness of the controller on the realistic testbed is mapped and appraised.

This paper strongly contributes to the viewpoint highlighted in [20], i.e. that fractional order (FO) calculus solutions facilitate better performance compared to the best achievable ones previously using integer-order calculus. We present rigorous study of fractional-order discrete-time linear PID-type control design for processes with input delay. The designed controllers are validated on the experimental water tank volume control testbed. A particular highlight of this paper is the implementation of the fractional-order controller on Programmable Logic Controller (PLC) for the experiment. As part of the latter, effects of the discrete-time approximation via trapezoidal (Tustin) rule and Backward (Euler) rule are appraised.

Advantages of fractional-order PID closed-loop feedback in comparison to integer-order PID control are illustrated via use of with quadratic cost 
functions and analysis in the frequency domain. Integer-order PID control is designed based on the same specifications. To show the effectiveness of fractional-order PID in improving stability margins, dynamic performance and reference tracking, both simulation-based and experimental tests on a water tank volume system (testbed) are performed.

\section{Fractional order integral-differential operators}

This section presents a brief introduction to fractional order methods and in particular integro-differential operators. In the general case a fractional order operator of a complex order $\alpha$, can be defined as [7]:

$$
t_{0} D_{t}^{\alpha}:= \begin{cases}\frac{d^{\alpha}}{d t^{\alpha}} & \operatorname{Re}(\alpha)>0 \\ 1 & \operatorname{Re}(\alpha)=0 \\ \int_{t_{0}}^{t}(d \tau)^{-\alpha} & \operatorname{Re}(\alpha)<0\end{cases}
$$

We assume that $\alpha \in \mathbb{R}_{+}$. In particular cases operator $t_{0} D_{t}^{\alpha}$ can be defined in several ways, see [13. From the control application point of view the most popular are definitions of the Riemann-Louville's and Caputo's fractional order integro-differential operators. Here we concentrate on the first one and its discretization. The Riemann-Louville (RL) fractional order $\alpha, \alpha \in[n-1, n), n \in \mathbb{N}$, integro-differential operator is defined as [3], [7], [15]

$$
{ }_{t_{0}}^{R L} D_{t}^{\alpha} f(t):=\frac{1}{\Gamma(n-\alpha)} \frac{d^{n}}{d t^{n}} \int_{t_{0}}^{t} \frac{f(\tau)}{(t-\tau)^{\alpha-n+1}} d \tau
$$

where $\Gamma$ is the Euler function, i.e. $\Gamma(z)=\int_{0}^{\infty} e^{-\tau} \tau^{z-1} d \tau$ with $\operatorname{Re}(z)>0$.

The Laplace transform of this operator is given as

$$
\mathcal{L}\left[{ }_{0}^{R L} D_{t}^{\alpha} f(t)\right]=s^{\alpha} F(s)-\left.\sum_{k=0}^{n-1} s^{k}\left[{ }_{0}^{R L} D_{t}^{\alpha-k-1} f(t)\right]\right|_{t=0}
$$

where $F(s)=\mathcal{L}[f(t)]$ with variable $s=j \omega$ and $s \in \mathbb{C}$, denotes the Laplace transform of the function $f(t)$.

In order to realize fractional order (FO) controllers perfectly, all the past input should be memorized. In practice this is not possible, hence approximation or discretization method should be used. The most commonly used discretization approach in fractional case is termed as short memory principle [11]. It is based on the fractional order Grünwald-Letnikov (GL) operator of order $\alpha \in(n-1, n)[1],[3]$, [15]:

$$
{ }_{t_{0}}^{G L} D_{t}^{\alpha} f(t):=\lim _{h \rightarrow 0} \frac{1}{h^{\alpha}} \sum_{j=0}^{\left[\frac{t-t_{0}}{h}\right]}(-1)^{j}\left(\begin{array}{c}
\alpha \\
j
\end{array}\right) f(t-j h)
$$


where $h>0$ and

$$
\left(\begin{array}{l}
\alpha \\
j
\end{array}\right)= \begin{cases}\frac{\alpha(\alpha-1) \ldots(\alpha-j+1)}{j !} & \text { for } j=1,2,3, \ldots \\
1 & \text { for } j=0\end{cases}
$$

denotes the binomial coefficient. Since values of the binomial coefficient near "starting point" $t_{0}=0$ are small enough to be neglected, see [11]. It is easy to note also that ${ }_{0}^{R L} D_{t}^{\alpha} f(t)={ }_{0}^{G L} D_{t}^{\alpha} f(t)$ for any continuously differentiable function $\mathrm{f}$. In the following, if it is just not misleading, we are going to skip symbols $R L$ or $G L$ in fractional order differential operators.

The discrete equivalent of the fractional-order differential operator $t_{0} D_{t}^{\alpha}$ is given by [11]

$$
\mathcal{Z}\left[D_{t}^{\alpha} f(t)\right]=h^{-\alpha} \sum_{j=0}^{m}(-1)^{j}\left(\begin{array}{c}
\alpha \\
j
\end{array}\right) z^{-j} F(z)
$$

where $F(z)=Z[f(t)], m=\left[\frac{L}{h}\right]$ where $L$ is the number of past values taken into account (so called memory of the system), and $z$ is the complex variable or shift operator $z^{-1}$.

For the purpose of carrying out numerical computation, formula 2.5 leads to Grünwald-Letnikov operator of the real function $f(t)$, the revised version of 2.4 is rewritten as

$$
{ }_{t_{0}} D_{t}^{\alpha} f(t)=\sum_{j=0}^{\frac{t-t_{0}}{h}} a_{j}^{(\alpha)} f(t-j h)
$$

with $\alpha_{j}^{(\alpha)}=\frac{(-1)^{j}}{h^{\alpha}}\left(\begin{array}{c}\alpha \\ j\end{array}\right)$, and $h$ is the step-size in computation [14].

\section{System modelling and identification}

Figure 1 illustrates the schematic diagram of the experimental setup that represents the process model with input delay characteristic, it consists the MPS water Compact Workstation developed by Festo, PLC Simatic controller developed by Siemens and PC computer with TIAportal + WinCC and Matlab/Simulink software. In addition, the considered water volume control loop includes ultrasonic fluid level sensor and controller with ana$\log$ and digital terminals. The major electrical signal connections, both for measurement and control, are presented by red-colored lines; the PC communication is shown via black-colored lines with serial port: RS-232.

During the open-loop identification, measurements were performed by the EasyportDA signal interface and FluidLab-PA. The water volume or water level is the sum of all water flowing into the system, while the outflow flow rate equals the inflow flow-rate. In our case the outflow is set to a 
constant value of $15 \%$. In particular, the greater the inflow the faster the level in the container increases per unit time. When the water tank is filling under a constant flow rate (inflow), the pressure of the water column at the bottom of the container is proportional to the level. The pressure at the bottom increases with level, which results to an increase in outflow until equilibrium (with the inflow) is reached (hence results to a constant level, i.e. no more increase reached "steady-state end value"). The step command signal of 10 voltage increases the speed of the pump from $0 \%$ up to $100 \%$, where the voltage control signal can change from 0 to $10 \mathrm{~V}$.

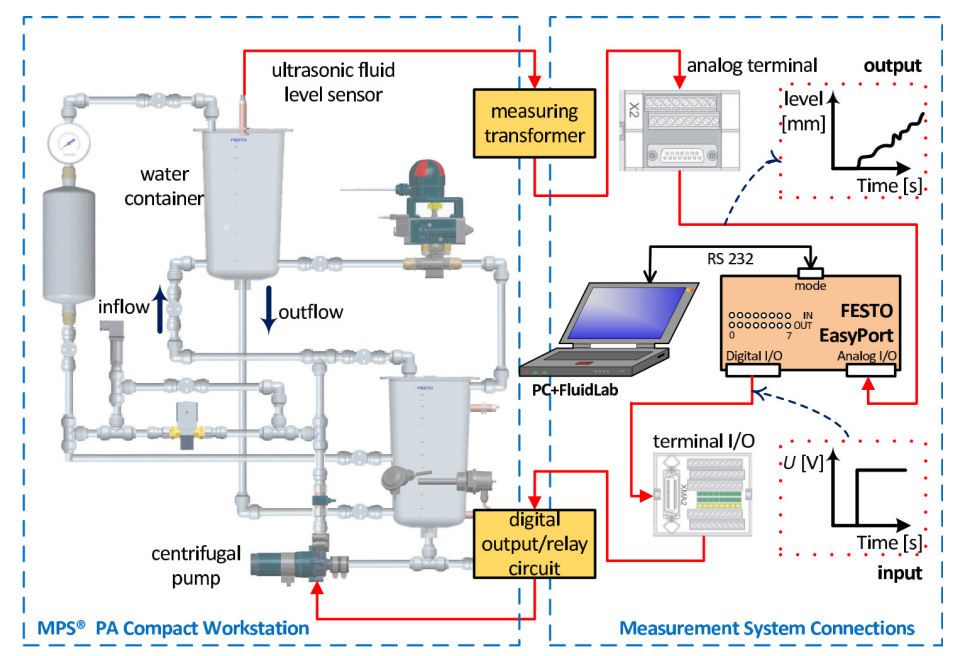

FiguRE 1. Diagram of the fluid volume controlled system

A discrete-time model of the open-loop system was identified based on the time-raw measured water volume data. The water tank experimental response for the step input signal of the pump is given in Figure 2. The sampling time used, and appropriate for such a slow dynamics system, is $0.15 \mathrm{~s}$. The model fit to estimation data was approximately $93.5 \%$. The identified continuous time model is given by $G_{0}(s)=\frac{l(s)}{u(s)}=\frac{1.6841}{1+1052.6 s} e^{-8.544 s}$, and (in continuous time). While the discrete-time version can be seen in (3.7), with the input delay evident from the relevant delay operator part.

$$
G_{0}(z)=\frac{l(z)}{u(z)}=z^{-57} \frac{9.6 e-06(z+24)}{z-1}
$$

Note that the time delay based on the system measurement in Figure 2 is approxmiately $3 \mathrm{~s}$, then after taking into account the power start-up pump delay and the A/D terminal delay, the total time delay of the system (3.7) is equal to $8.544 \mathrm{~s}$. The state $l$ represents water volume and $u$ characterised 


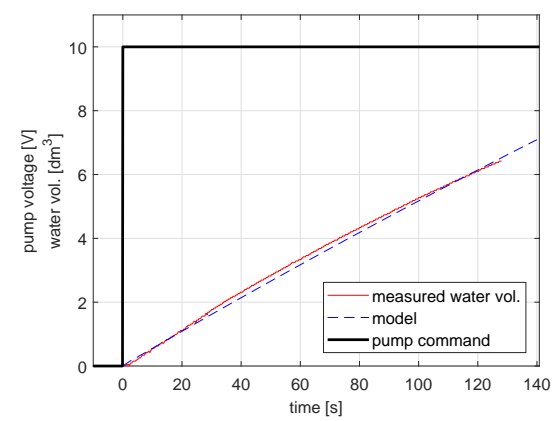

(a) Full picture of time response

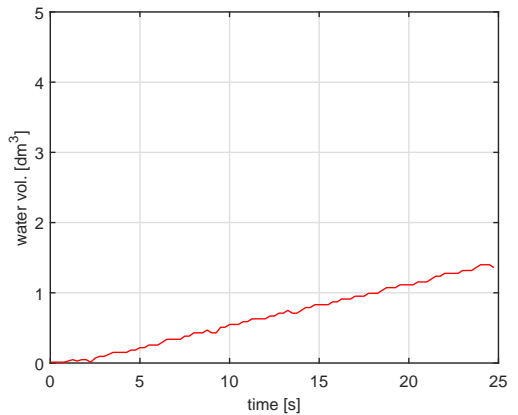

(b) water volume - time zoom

Figure 2. Model dynamics identification

the controller output (pump effort) in the range 0\%-100\%. The identified model represents a good approximation of the process under investigation, however we expect a level of robustness to be enabled by the designed controller.

\section{Fractional order controller design}

A continuous time linear input-output system can be described as linear fractional differential equation of order $\delta, \delta>0$, of one (time) variable:

$$
\sum_{i=0}^{n} a_{i 0} D_{t}^{\alpha_{i}} y(t)=\sum_{k-0}^{m} b_{k} D_{t}^{\beta_{i}} u(t)
$$

where $\alpha_{n}>\alpha_{n-1}>\cdots>\alpha_{1}>\alpha_{1} \geq 0, \beta_{m}>\beta_{m-1}>\cdots>\beta_{1}>\beta_{0} \geq 0$ are real numbers, $a_{i}, b_{k} \in \mathbb{R} i=0,1, \ldots, n, k=0,1, \ldots, m$. The transfer function of the considerate system is of the form:

$$
G(s)=\frac{b_{m} s^{\beta_{m}}+b_{m-1} s^{\beta_{m-1}}+\cdots+b_{0} s^{\beta_{0}}}{a_{n} s^{\alpha_{n}}+a_{n-1} s^{\alpha_{n-1}}+\cdots+a_{0} s^{\alpha_{0}}}
$$

We assume that the system (4.8) is of the commensurate order, that is all orders of derivation in (4.8) are integer multiplies of a base order $\alpha, \alpha \in \mathbb{R}_{+}$. Then, substituting $\lambda=s^{\alpha}$, the transfer function (4.9) of the system (4.9) with respect to zero initial conditions can be written in the form:

$$
\tilde{G}(\lambda)=\frac{b_{m} \lambda^{m}+b_{m-1} \lambda^{m-1}+\cdots+b_{1} \lambda+b_{0}}{a_{n} \lambda^{n}+a_{n-1} \lambda^{n-1}+\cdots+a_{1} \lambda+a_{0}}
$$


Hence, taking into account again that $\lambda=s^{\alpha}$ we obtain:

$$
G(s)=K \sum_{i=1}^{n} \frac{A_{i}}{s^{\alpha}+\lambda_{i}}
$$

with constant coefficients $K, A_{i}, i=1,2, \ldots, n$.

\subsection{Conventional vs Fractional order controller.}

$$
K_{c-p i d}(s)=k_{p}+\frac{k_{i}}{s}+k_{d} s
$$

with frequency response, $(s=j \omega), K_{p i d}(j \omega)=k_{p}-j \frac{k_{i}}{\omega}+k_{d} j \omega$. The frequency response yields the following magnitude (linear) and phase,

$$
\begin{aligned}
& \left|K_{c-p i d}(j \omega)\right|=\sqrt{k_{p}^{2}+\left(k_{d} \omega-\frac{k_{i}}{\omega}\right)^{2}}, \\
& \angle K_{c-p i d}(j \omega)=\tan ^{-1}\left(\frac{k_{d} \omega-\frac{k_{i}}{\omega}}{k_{p}}\right)
\end{aligned}
$$

Contrary to the conventional PID controller, the fractional equivalent, i.e. by $P I^{\lambda} D^{\mu}$, is given by

$$
C_{f-p i d}(s)=k_{p}+\frac{k_{i}}{s^{\lambda}}+k_{d} s^{\mu}=\frac{k_{p} s^{\lambda}+k_{i}+k_{d} s^{(\lambda+\mu)}}{s^{\lambda}}
$$

where $\lambda$ and $\mu \in \mathbb{R}_{+}$, are either integer or non-integer, thus the FO controller 4.15 has five parameters to tune and this is a more challenging design exercise to perform compared to the conventional integer-order PID controller [12], [15], 17]. Note that gains $k_{p}, k_{i}, k_{d}$ in the FO PID do not necessarily have the same values as in the conventional PID controller (and this is made clear by use of "integer" and "fractional" prefixes).

The model for control design (3.7) presented earlier in continuous-time is in the typical form of a first-order plus time delay transfer function, i.e.

$$
G(s)=\frac{k}{T s+1} e^{-T_{0} s}
$$

where $k$ denotes a static gain, $T$ is a time constant, and $T_{0}$ is the input time delay. The modulus and phase are $|G(j \omega)|=\frac{k}{\sqrt{1+\omega^{2} T^{2}}}, \arg \{G(j \omega)\}=$ $-\arctan \left(\frac{\omega}{T}\right)-\omega T_{0}$, respectively. 
The open-loop transfer function is listed for the integer-order PID, i.e. $L_{c-p i d}(s)=G(s) K_{c-p i d}(s)$, with modulus and argument as follows

$$
\begin{aligned}
\left|L_{c-p i d}(j \omega)\right| & =\frac{k}{\sqrt{1+\omega^{2} T^{2}}} \sqrt{k_{p}^{2}+\left(k_{d} \omega-k_{i} \omega^{-1}\right)^{2}} \\
\angle L_{c-p i d}(j \omega) & =\angle G(j \omega)+\angle K_{c-p i d}(j \omega) \\
& =-\omega T_{0}-\arctan \left\{\frac{\frac{\omega}{T}+k_{p}^{-1}\left(-k_{d} \omega+k_{i} \omega^{-1}\right)}{1-\frac{\omega}{T} k_{p}^{-1}\left(-k_{d} \omega+k_{i} \omega^{-1}\right)}\right\}
\end{aligned}
$$

While in the fractional-order case, with open loop $L_{f-p i d}(s)=G(s) C_{f-p i d}(s)$, the modulus and phase are given by

$$
\left|L_{f-p i d}(j \omega)\right|=\frac{k}{\sqrt{1+\omega^{2} T^{2}}} \sqrt{\operatorname{expr} 1 a}=\frac{k}{\sqrt{1+\omega^{2} T^{2}}} \sqrt{\operatorname{expr} 1 b}
$$

where,

$$
\begin{aligned}
& \operatorname{expr} 1 \mathrm{a}=\left(k_{p}+k_{i} \omega^{-\lambda} \cos \left(\frac{\lambda \pi}{2}\right)+k_{d} \omega^{\mu} \cos \left(\frac{\mu \pi}{2}\right)\right)^{2} \\
& +\left(-k_{i} \omega^{-\lambda} \sin \left(\frac{\lambda \pi}{2}\right)+k_{d} \omega^{\mu} \sin \left(\frac{\mu \pi}{2}\right)\right)^{2} \\
& \operatorname{expr} 1 \mathrm{~b}=k_{p}^{2}+k_{i}^{2} \omega^{-2 \lambda}+k_{d}^{2} \omega^{2 \mu}+2 k_{p}\left(k_{d} \omega^{\mu} \cos \left(\frac{\mu \pi}{2}\right)+k_{i} \omega^{-\lambda} \cos \left(\frac{\lambda \pi}{2}\right)\right) \\
& +2 k_{i} k_{d} \omega^{(-\lambda+\mu)} \cos \left(\frac{(\mu+\lambda) \pi}{2}\right) \\
& \angle L_{f-p i d}(j \omega)=\angle G(j \omega)+\angle K_{f-p i d}(j \omega) \\
& =-\omega T_{0}-\arctan \left(\frac{\omega}{T}\right)-\tan \left\{\frac{-k_{i} \omega^{-\lambda} \sin \left(\frac{\lambda \pi}{2}\right)+k_{d} \omega^{\mu} \sin \left(\frac{\mu \pi}{2}\right)}{k_{p}+k_{i} \omega^{-\lambda} \cos \left(\frac{\lambda \pi}{2}\right)+k_{d} \omega^{\mu} \cos \left(\frac{\mu \pi}{2}\right)}\right\}
\end{aligned}
$$

Clearly, the introduced degree of freedom in loop shaping by the fractionalorder equivalent can be seen from the above relationships. Now, it is well known that selecting Bode's ideal transfer function for the desired loop transfer function

$$
L_{B}(s)=\left(\frac{\omega_{g}}{s}\right)^{\alpha}
$$

facilitates closed-loop insensitivity to gain changes [21]. In (4.19), $\omega_{g}$ is the desired gain crossover frequency (i.e.where $\left|L\left(j \omega_{g}\right)\right|=1$ ) (in linear magnitude terms) and $\alpha \in \mathbb{R}_{+}$, the latter parameter being of paramount importance regarding the loop transfer function magnitude response slope 


\section{PLC-BASED DISCRETE FRACTIONAL-ORDER CONTROL}

properties near crossover The modulus and phase of the aforementioned ideal loop transfer function are $\left(\frac{\omega_{g}}{\omega}\right)^{\alpha}$ and $-\alpha \frac{\pi}{2}$, respectively.

The actual loop transfer function for this system with the FOPID is

$$
L_{f-p i d}(s)=\left(\frac{k}{T s+1}\right) \frac{k_{p} s^{\lambda}+k_{i}+k_{d} s^{(\lambda+\mu)}}{s^{\lambda}} e^{-T_{0} s}
$$

Assuming complementary integrator/differentiator orders, i.e. $\mu=1-\lambda$,

$$
L_{f-p i d}(s)=\left(\frac{k}{T s+1}\right) \frac{k_{p} s^{\lambda}+k_{i}+k_{d} s}{s^{\lambda}} e^{-T_{0} s}
$$

Hence enabling only fractional integrator contribution. One can clearly see the constraint introduced by the input delay portion (essentially in phase margin achievement normally and in closed-loop bandwidth, i.e. speed of response, aspects). In order to obtain the open-loop transfer function as Bode's ideal transfer function expect time delay the controller must obey:

$$
K(s)=K_{p}\left((T s+1) / s^{\lambda}\right)=K_{p}\left(1 / s^{\lambda}+T s^{(1-\lambda)}\right)
$$

We can observe that controller 4.22 is the ideal form of (4.15). Moreover, in the special case of: $k_{d}=T K_{p}, k_{i}=K_{p}$, and $k_{p} \ll K_{p}$, one gets

$$
K_{p}\left(1 / s^{\lambda}+T s^{(1-\lambda)}\right) \cong k_{p}+k_{i} / s^{\lambda}+k_{d} s^{(1-\lambda)}
$$

Hence, the following considerations are based on this assumption, with the actual open-loop transfer function simplifying to:

$$
L_{A}(s)=\left(\left(k K_{p}\right) s^{-\lambda}\right) e^{-T_{0} s}=\left(\sqrt[\lambda]{k K_{p}} / s\right)^{\lambda}\left(e^{-T_{0} s}\right)
$$

Note that equation (4.24) is a "delayed" form of (4.19). In addition, recall that the phase margin for the ideal transfer function in (4.19) is $\phi_{m}=$ $(1-\lambda / 2) \pi$. Referring to the actual loop transfer function from (4.24), fractional-order $\lambda$ can be further investigated in the framework of loop shape design. Given that $(j \omega)^{\lambda}=|\omega|^{\lambda} e^{j \lambda \pi / 2}$, the gain and phase of 4.24 are:

$$
\left|L_{A}(j \omega)\right|=\left(k K_{p}\right) \omega^{-\lambda}, \quad \phi_{L_{A}}(\omega)=\arg \left(L_{A}(j \omega)\right)=-T_{0} \omega-\lambda \pi / 2
$$

Also, crossover frequencies $\omega_{g}$ (i.e. gain) and $\omega_{p}$ (i.e. phase) are given by:

$$
\left|L_{A}\left(j \omega_{g}\right)\right|=1, \quad \phi\left(\omega_{p}\right)=\arg \left(L_{A}\left(j \omega_{p}\right)\right)=-\pi
$$

Now, assuming $A_{m}$ a given gain margin (GM) and $\phi_{m}$ a given phase margin (PM) one can get:

$$
\left(k K_{p}\right) \omega_{p}^{-\lambda}=1 / A_{m}, \quad \phi_{m}=\pi(1-\lambda / 2)-T_{0} \omega_{g}
$$

Whereby $\omega_{p}=\pi T_{0}^{-1}(1-\lambda / 2)$. The above equations enable flexibility in selecting the order $\lambda$ based on loop shaping characteristics (although major 
ones can be chosen and the design to be based on in the sequence). Major aspects here are GM and PM, while note that the two crossover frequencies are interlinked via the above equations (once a PM is chosen). Typical engineering based choices for the margins are normally GM of 4-6 $\mathrm{dB}$ and PM of 45-85 degrees.

\section{Discrete fractional order controller realization}

In reference [8] authors based on the classical forward difference operator $\left(\Delta_{h} x\right)(t)=\frac{x(t+h)-x(t)}{h}$ introduced discrete Riemann-Liouville-type fractional operator. In order to define this operator, firstly note that:

$$
\left(\Delta_{h}^{x}\right)(t):=\left(\Delta_{h} x \circ \Delta_{h} x \circ \cdots \circ \Delta_{h} x\right)(t)=h^{-n} \sum_{j=0}^{n}(-1)^{n-k}\left(\begin{array}{l}
n \\
j
\end{array}\right) x(t+j h)
$$

Then, the fractional h-sum of order $\alpha, \alpha>0$, is defined by

$$
\left({ }_{a} \Delta_{h}^{-\alpha} x\right)(t):=h^{\alpha} \sum_{j=0}^{n}\left(\begin{array}{c}
n-j+\alpha-1 \\
n-j
\end{array}\right) x(a+j h)
$$

for any $n \in N_{0}$. The Riemann-Liouville-type fractional difference operator ${ }_{a} \Delta_{h}^{\alpha}$ for a function $x:(h N)_{a} \rightarrow R$ is given by [8]

$$
\left({ }_{a} \Delta_{h}^{\alpha} x\right)(t):=\left(\Delta_{h}\left({ }_{a} \Delta_{h}^{-(1-\alpha)} x\right)\right)(t)
$$

where $t \in(h N)_{a+(1-\alpha) h}$. In [2] was shown that if $a=(\alpha-1) h$ then

$$
\left({ }_{a=0} \widehat{\Delta}_{h}^{\alpha} y\right)(t+a)=\left({ }_{a} \Delta_{h}^{\alpha} x\right)(t)
$$

where $x(t)=y(t-a)$ for any $t \in(h N)_{a}$. The discrete-time control law of the controller 4.15 need to express by the difference operator from equation (5.28). Then, the obtained control laws can be written as [14]:

$$
u(t)=\left(k_{p}+k_{i t_{0}} D_{t}^{-\lambda}+k_{d t_{0}} D_{t}^{\mu}\right) e(t)
$$

where $e(t)$ is control error and $t_{0} D_{t}^{\mu}$ is the fractional order differential. Approximation of this operator by Grunvald-Letnikov-type operator $t_{0} \widehat{\Delta}_{h}^{\alpha}$ leads to transformation [9]:

$$
{ }_{t_{0}} D_{t}^{\alpha} e(t)={ }_{t_{0}} \widehat{\Delta}_{h}^{\alpha} e(t):=\sum_{j=0}^{N-1} a_{j}^{(\alpha)} e(t-j h)
$$

where $h=\frac{\left(t-t_{0}\right)}{N}$ is a step width, $t$ denotes an interval for fractional order discretization computations, $t_{0}$ is an initial time and $N$ is an amount of function's discretized point. In our considerations we assume that $t_{0}=0$. 
5.1. Discrete PSE approximation and PLC implementation. In order to implement the operator (2.1) in the PLC, the discrete approximation method is required. The most known method of PSE (Power Series Expansion) and CFE (Continuous Fractional Expansions) can be applied. These methods allow us to estimate a fractional order terms with the use of digital FIR or IIR filter. Major advantage of the PSE is that this approximant bases directly on discrete version of the GL operator (2.4), and approximation has the form of polynomials, that is, the discretized fractional operator has the form of a FIR filter containing only zeros. Its numerical realization of the discrete implementation requires a relative long memory buffer in order to obtain a good control quality. The CFE approximation has the form of n-th order IIR filter containing both zeros and poles, cause uses the trapezoidal integration rule. Thus its realization is easier since obtained approximation order is relatively low. However, the CFE-based method can cause numerical problems ending up with a low upper limit of implementation length for CFE approximator.

The most straightforward method to discrete-time approximation of fractional order function is the direct discretization using finite memory length based on definition (2.1). In this method, the discretization of fractional order differentiator/integrator $s^{ \pm \alpha}$ is expressed by the discrete equivalent $s=\omega\left(z^{-1}\right)$ with the shift operator $z^{-1}$. In case of the backward fractional difference rule, $\omega\left(z^{-1}\right)=\left(1-z^{-1}\right)$, and applying the PSE to the $\left(1-z^{-1}\right)^{\alpha}$, we obtain formula $(2.1)$ for the fractional derivative of order $\alpha$.

Using PSE, the discrete equivalent of the fractional order operator, for the transfer function $G(z)=\frac{Y(z)}{F(z)}$, is given by

$$
D^{ \pm \alpha}(z)=G(z)=h^{\mp \alpha} \operatorname{PSE}\left\{\left(1-z^{-1}\right)^{\mp \alpha}\right\} \simeq h^{\mp \alpha} P_{p}\left(z^{-1}\right)
$$

where $Y(z)$ and $F(z)$ are the $Z$ transforms of the output $y(j h)$ and input $f(j h)$ sequences. Based on the short memory principle [?], the discrete approximation of the fractional order integral/differential operator $\left(\omega\left(z^{-1}\right)\right)^{ \pm \alpha}$, is given by

$$
D^{ \pm \alpha}(z)=\left(\omega\left(z^{-1}\right)\right)^{ \pm \alpha}=h^{\mp \alpha} z^{-[L / h]} \sum_{j=0}^{[L / h]}(-1)^{j}\left(\begin{array}{c} 
\pm \alpha \\
j
\end{array}\right) z^{[L / h]-j}
$$

where $L$ is the memory length, and $(-1)^{j}\left(\begin{array}{c} \pm \alpha \\ j\end{array}\right)$ are the binomial coefficients according to relation 2.4. Second discrete-time approximation, of the PSE scheme, is the use of the trapezoidal rule, called the Tustin method, in which, discretization of the differentiator/integrator is expressed by the generating function

$$
\omega\left(z^{-1}\right)=2 \frac{1-z^{-1}}{1+z^{-1}}
$$


The PSE both Euler and Tustin methods are used with approximation order of 5 to obtain discrete fractional order PID controller (DFOPID) [14]. Table 1 presents different gain/phase margins and fractional orders for the fractional order discrete representation of the DFOPID controllers used in the simulations and experimental investigations. The FOPID controller

TABLE 1. Various GM and PM specifications for the DFOPID $\left(T_{\mathrm{s}}=0.15 \mathrm{~s}\right)$

\begin{tabular}{c|ccccc}
\hline $\begin{array}{c}\text { Approx. method } \\
\text { (DT integration) }\end{array}$ & $\begin{array}{c}\text { GM } \\
\mathbf{A}_{\mathbf{m}}\end{array}$ & $\begin{array}{c}\text { PM } \\
\phi_{\mathbf{m}}(\mathbf{d e g})\end{array}$ & $\begin{array}{c}\text { FO } \\
\lambda\end{array}$ & $\begin{array}{c}\text { FO } \\
\mu\end{array}$ & $\begin{array}{c}\text { DFOPID } \\
\text { id }\end{array}$ \\
\hline \hline & 4 & 65 & 1.02 & -0.02 & niPID1 \\
PSE of Euler rule or & 4 & 75 & 0.92 & 0.08 & niPID2 \\
PSE of Tustin rule & 5 & 85 & 0.87 & 0.13 & niPID3 \\
& 6 & 85 & 0.90 & 0.10 & niPID4 \\
\hline
\end{tabular}

constant gains were tuned, by solving equations for the gain and phase crossover frequency and GM/PM specifications given in Section 4. Then, these parameters are recalculated according to the FOPID discrete approximation, PLC implementation and the Festo MPS specification. Particularly, the inherent gains of the PLC A/C and C/A converters equal to 1/10 $\mathrm{V}$. In the Festo MPS system the ultrasonic sensor level/voltage gain is $1 / 22$ $(10 \mathrm{~V}$ for $220 \mathrm{~mm})$. The measurement noise is about $0.1 \mathrm{~V}$ peak to peak. Additionally, the constant gains are finally tuned during the experiments, thus, many sets of the values are tested. The optimized gains of the FOPID controller 4.15), which also meet the relation (4.23) are: $k_{d}=T K_{p}=7.5$, $k_{i}=K_{p}=50$, and $k_{p}=0.68$, where the sampling time $T_{\mathrm{s}}=0.15 \mathrm{~s}$. The total feedback gain, including $\mathrm{A} / \mathrm{C}$ and $\mathrm{C} / \mathrm{A}$ converters and sensor level gains, is equal to $220 k_{p}$. The FOPID gains are constant for all simulations and experiments in this paper.

PLC implementation of the DFOPID algorithm is performed via guidelines formulated in the IEC 61131 standards. The program structure of the PLC controller S7-300 (Simatic of Siemens) contains the organization blocks (OB1) which determine and call the function blocks (FB) and the control functions (FC). These have access to the function process data collected in the data blocks (DB). The fractional-order controller code is represented by the FB which have access to the inherent memory of the DB. The FB with DFOPID controller is called in an OB35 block (a cyclic interrupt activated periodically each desired sampling time $6 \mathrm{~ms}$ or 150 $\mathrm{ms}$ ). Other parts of the PLC program (communication between the PC and the PLC, management, sensors, etc.) are gathered into the OB1 block. 


\section{PLC-BASED DISCRETE FRACTIONAL-ORDER CONTROL 13}

The FOPID controller algorithm is implemented in the structural control language (SCL), which is a high-level textual programming language based on PLCs standard. SCL conforms to the IEC 113-3 standard and is sufficient for numerical calculations with exponential operators, times/counter functions and control structures, i.e. loops (FOR/WHILE/REPEAT) and alternatives (IF THEN/CASE/GOTO). See Figure 3 for the sample of SCL algorithm including the FOPID terms with the PSE approximation. During

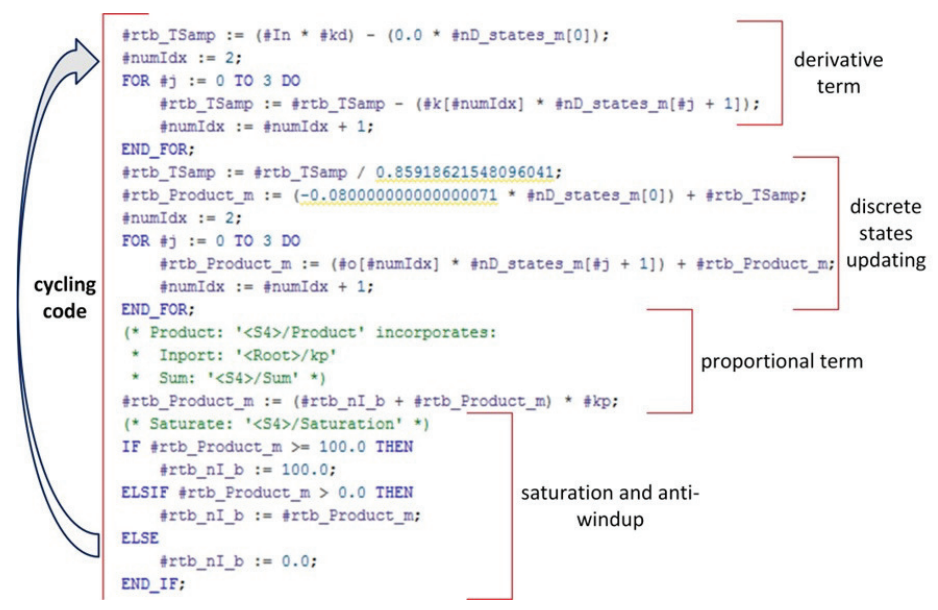

FiguRE 3. DFOPID code on the S7-300 PLC controller

PLC implementation of the control system, physical limits of the process control should be considered. The DFOPID controller output is processed via saturation block in the range $0-10 \mathrm{~V}$. Also, in order to prevent integration wind-up in the controller when the pump is saturated, anti-windup filter is used (see code in Figure 3). The anti-windup scheme comprises back-calculation and clamping as well as a tracking mode to handle complex scenaria.

\section{Matlab simulation (Simulink) runs}

This section presents Matlab (Simulink) results for the water tank volume system controlled with DFOPID feedback loop prior to the experimental tests. Non integer terms are approximated via CRONE] formula [18. Both PSE (Euler and Tustin) discrete-time approximations are applied. The control system setup in Simulink is seen on Figure 5. In numerical calculations, given in Figure 5(a), the water tank volume system representation includes control model (3.7) and discrete representations of the

(fr. Commande Robuste dOrdre Non Entier) 


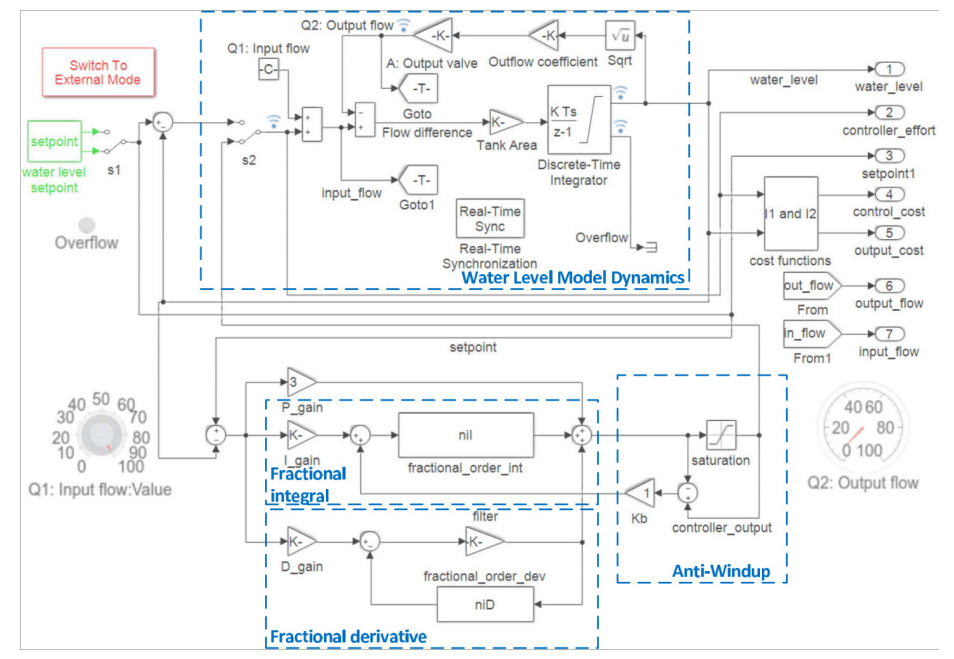

Figure 4. Fluid volume control system setup in Simulink

FOPID controllers (given in Table 1). In results of the computer simulation the step responses of the closed-loop systems with different parameters of the fractional-order controller are obtained. The rise time is increasing with PM increasing from $75 \mathrm{deg}$ to $85 \mathrm{deg}$, respectively. The poles distributions of the water volume closed-loop DFOPID system for different PM, and two discrete approximation methods, are given in Figure 6.

A number of system poles presented in Figure 6 are collected in Table 2. Particularly, only the dominant poles are presented in the map shown in Figure 6. The system with $\phi_{m}=65 \mathrm{deg}$ has poles located on the unit circle border $(1, \mathrm{j} 0)$, and cause the system output being not robust to input variations. Note that poles distribution and stability margin, itself, depends on the approximation method for discrete integration.

TABle 2. No. of poles for different DT approximation order

\begin{tabular}{c|cccc}
\hline $\begin{array}{c}\text { Approx. method } \\
\text { (DT integration) }\end{array}$ & $\begin{array}{c}\text { OL sys. } \\
\text { poles no. }\end{array}$ & $\begin{array}{c}\text { FO integr. } \\
\text { poles no. }\end{array}$ & $\begin{array}{c}\text { FO deriv. } \\
\text { poles no. }\end{array}$ & $\begin{array}{c}\text { CL sys. } \\
\text { poles no. }\end{array}$ \\
\hline \hline PSE of Euler rule or & 4 & 5 & 5 & 14 \\
PSE of Tustin & 4 & 3 & 3 & 10 \\
\hline
\end{tabular}

\section{Experimental testbed results}

In this section a series of experiments that appraise performance of the integer-order discrete PID controller to a set of the DFOPID controllers 


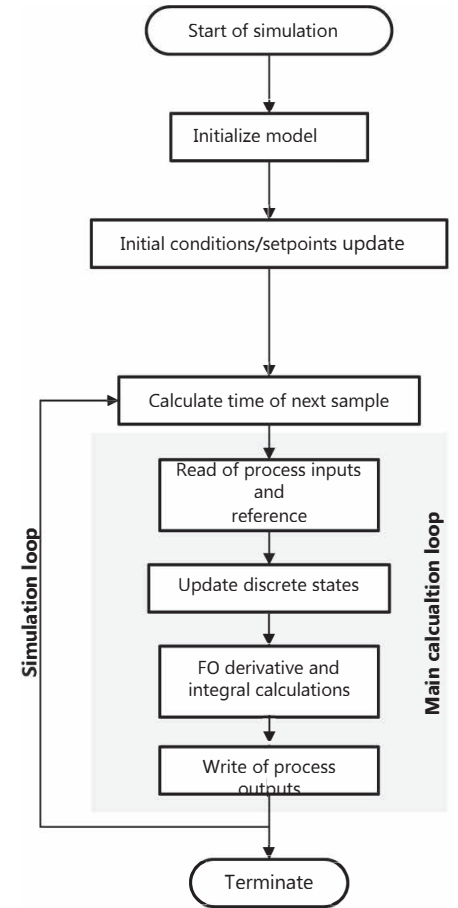

(a) Flow diagram (based on Sfunction)
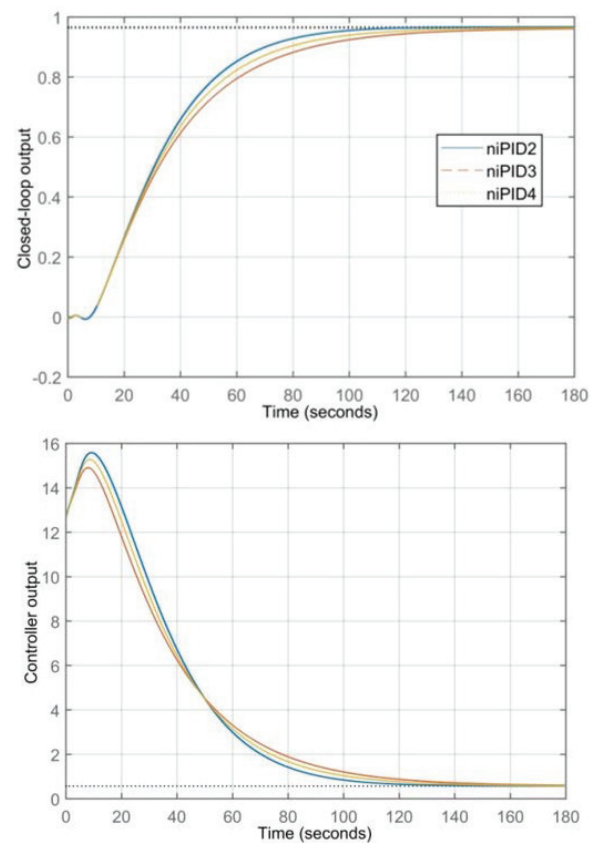

(b) Step response

FiguRE 5. Simulation algorithm and step responses of the fractional order system $\left(T_{\mathrm{s}}=0.15 \mathrm{~s}\right)$

obtained for the different fractional-orders are given. The experimental setup and PLC-based control configuration are presented in Figure 7, while the closed-loop system configuration is depicted on Figure 8 The relative optimal DFOPID controller is achieved to ensure desired system stability margin and obtain tracking to set-point changes. It is noted that the tuned controller parameters, satisfying the requirements given in Section 4, are the same for both the discrete PID and DFOPID controllers. The DFOPID closed-loop control system maintains desired dynamic performance, i.e. small oscillation and settling time, and the overshoot of $5 \%$ whereas the set-point change equals $100 \%$. Figure 9 presents the transient responses of the optimized process output variable and integer-order discrete PID controller output to the step set-point. Next, dynamic performance and stability of the integer-order discrete PID controller are compared with the DFOPID controller obtained for different $\lambda$ and $\mu$. 


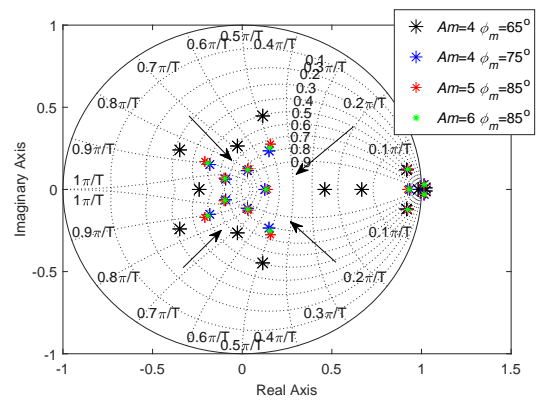

(a) Euler- 5th order

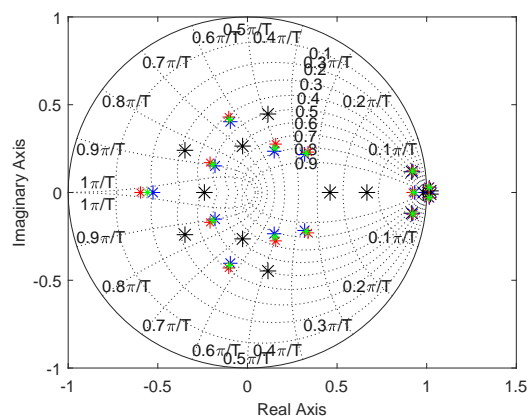

(c) Tustin- 5th order

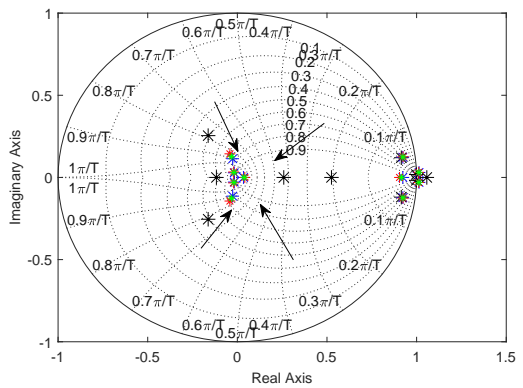

(b) Euler- 3rd order

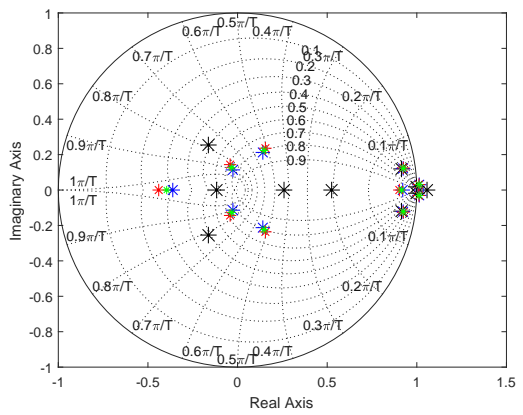

(d) Tustin- 3rd order

Figure 6. Pole locations for different approximation order of discrete controllers realization

Figure 9 presents the set-point responses of the process outputs, integerand non-integer-order discrete PID controllers outputs when the different sampling time is used for discrete controllers realizations, respectively. Figures show the closed-loop systems responses for the step reference, and controllers outputs, when the sampling time $T_{\mathrm{S}}$ is $0.15 \mathrm{~s}$ and $0.006 \mathrm{~s}$, respectively. The controller output is scaled to $0 \%-100 \%$, hence when the error between the set-point and the process output is negative the controller output is zero. In this situation, the proportional action is negative and the integral action takes negative values. In order to revert the integrator output to zero, the control error must be positive for a certain period. Also, the integration action takes additional values in case of controller output saturation in the anti-windup filter. Figures 9(c) and 9(d) illustrate clearly that in case of niPID1 (with the smallest GM and PM and discretisation setup scenario) for $T_{\mathrm{s}}=0.006 \mathrm{~s}$, the system output is unstable. Therefore, in this case the system is not robust to gain variations. 


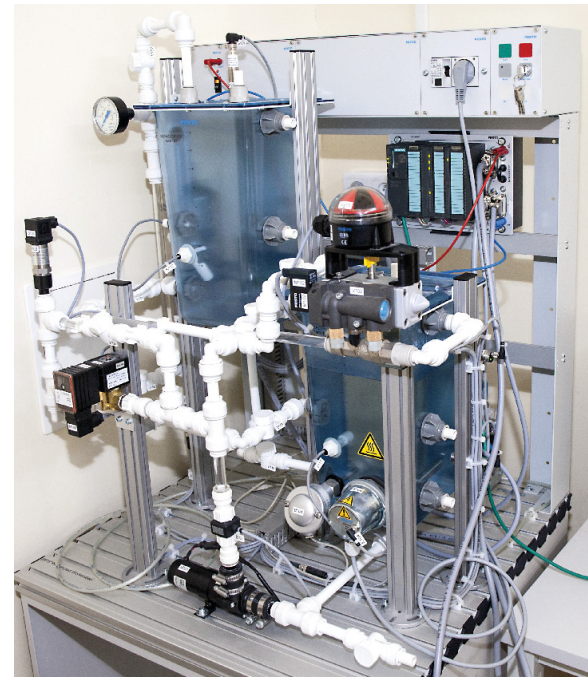

(a) The experimental setup

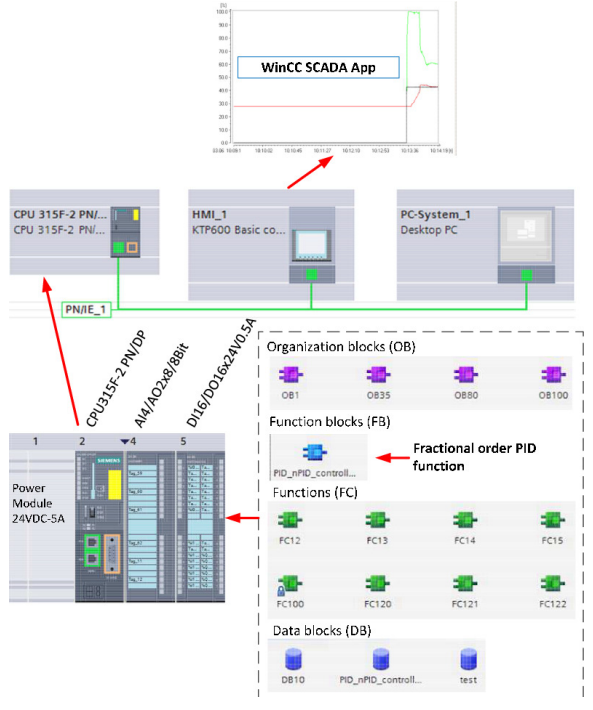

(b) $\mathrm{PLC} / \mathrm{HMI} / \mathrm{PC}$ station configuration

FiguRE 7. Experimental water tank testbed

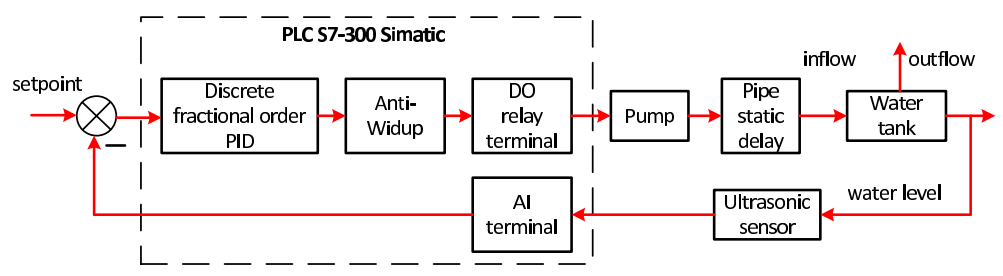

Figure 8. Closed-loop configuration setup

In addition, we appraise system outputs (for the stable version of niPID1) for different approximation methods for discrete-time (DT) fractional-order realization of the differentiator/integrator using mixed scheme of the backward difference (Euler) rule and the trapezoidal (Tustin) rule in Figure 10 . One may observe that, the calculations given at every step-size are shifted, which is a result of the difference in the numerical integration scheme used in the Euler and Tustin method respectively.

\section{Performance comparison of integer- and fractional-order setup}

As a final technical section we appraise both integer- and fractionalorder PID discrete control systems via quadratic cost functions of controller 


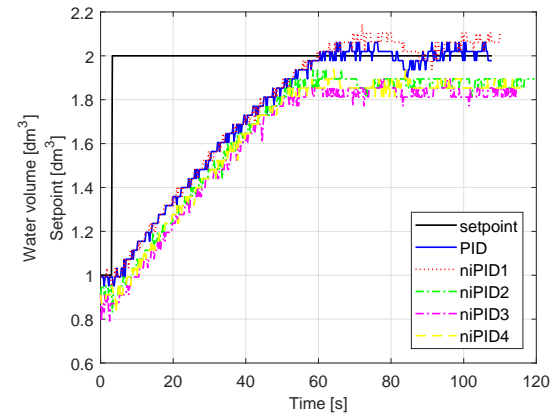

(a) Water volume (output)

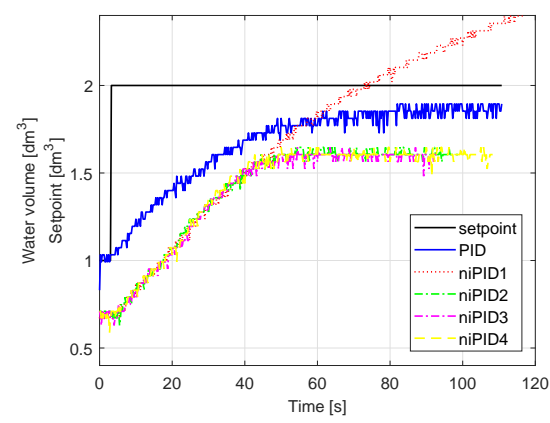

(c) Water volume (output)

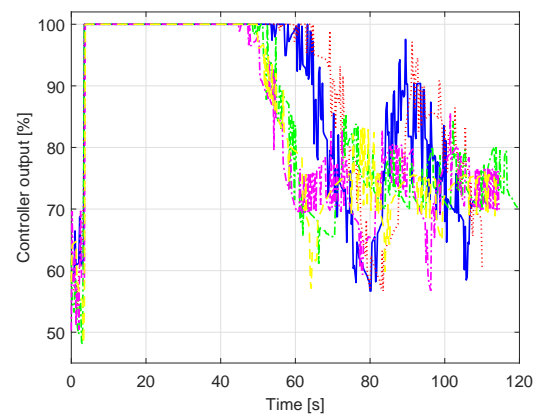

(b) Control signal

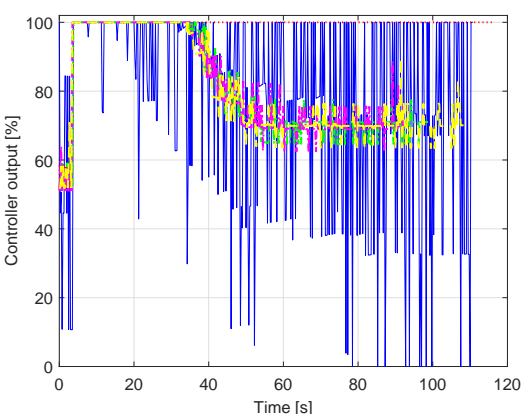

(d) Control signal

Figure 9. System response for DT controllers PSE (Euler rule) $T_{\mathrm{S}}=0.15 \mathrm{~s}$ (top set); $T_{\mathrm{s}}=0.006 \mathrm{~s}$ (bottom set)

outputs $u$, and of control error $e(t)=r(t)-l(t)$, where $r(t)$ is the set-point, i.e.:

$$
J_{1}=\int_{0}^{\infty}\left(u^{2}\right) d t ; \quad J_{2}=\int_{0}^{\infty}\left(e^{2}\right) d t
$$

The cost functions 8.37) are performed using cumulative numerical integration method and the comparison results are shown in Figure 11. The niPID3 associated system shows the better control performance. As expected, with reduced system stability margins the total energy of the controller output is higher compared to that for controllers referring to more stable margins.

Next, comparison of the integer- and non-integer PID discrete control closed-loop systems is given in the frequency domain. The discrete-time 


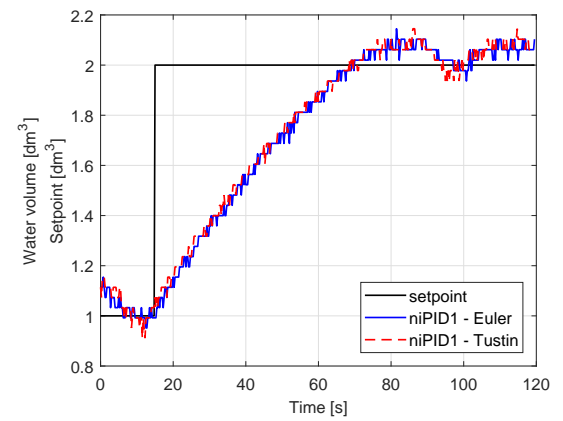

(a) Water volume (output)

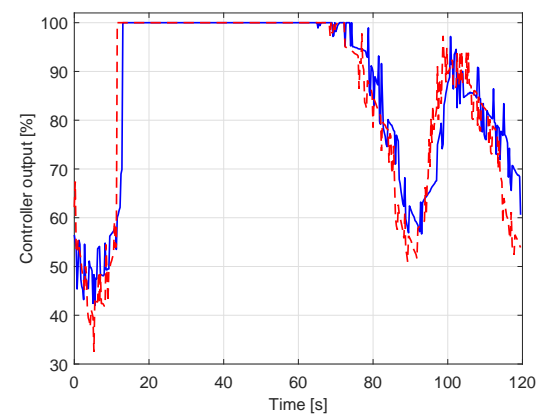

(b) Control signal

Figure 10. Appraising Euler and Tustin rule for DT controller realization $\left(\mathrm{niPID} 1 ; T_{\mathrm{s}}=0.15 \mathrm{~s}\right)$

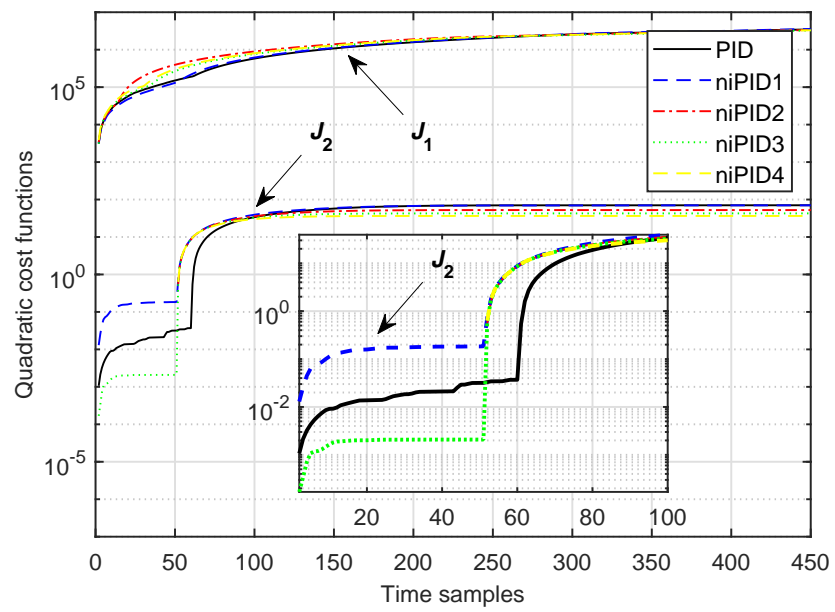

Figure 11. Quadratic cost function based comparison $\left(T_{\mathrm{s}}=0.15 \mathrm{~s}\right)$

version of the identified true model (7) together with the feedback controller niPID1,.., niPID4 is compared with the integer-order PID closedloop system. Figure 12(a) shows the closed-loop system frequency response. Particularly, the singular value plot is calculated for $G_{c l}\left(e^{\left(j \omega T_{\mathrm{s}}\right)}\right)$, where, in the case of the fractional-order system, $G_{c l}(z)=\left(K_{(f-P I D)}(z) G_{0}(z)\right) /(1+$ $\left.K_{(f-P I D)}(z) G_{0}(z)\right)$ is the discrete transfer function of the closed-loop system, $T_{\mathrm{s}}$ is the sampling time, and $\omega$ is frequencies between 0 and the Nyquist frequency of $\pi / T_{\mathrm{S}}$. Robustness of the integer- and fractional-order feed- 


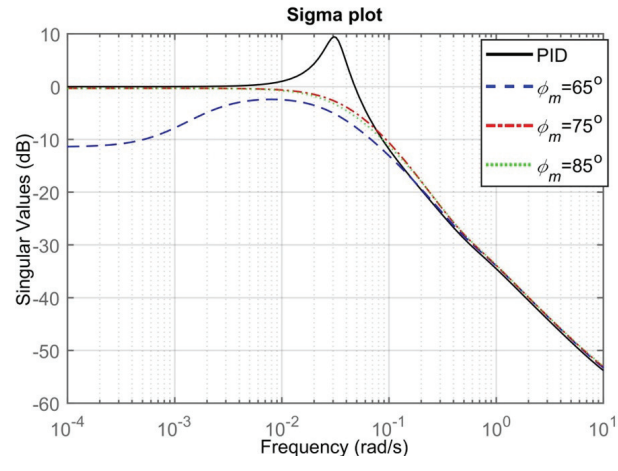

(a) Complementary sensitivity

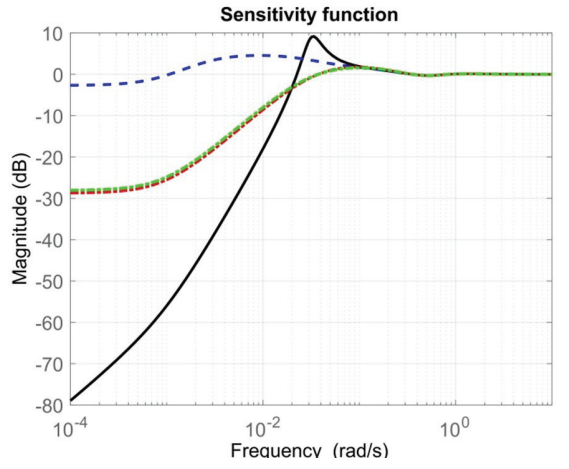

(b) Sensitivity

FiguRE 12. Frequency response system robustness appraisal $\left(T_{\mathrm{s}}=0.15 \mathrm{~s}\right)$

back loop systems is further investigated via sensitivity magnitude (singular value) plot seen on Figure 12(b). In the case of the non-integer-order setup, the sensitivity function is referred to as $S_{f r a c}=\left(I+L_{(f-p i d)}\right)^{(-1)}$, and the complementary sensitivity function, $T_{\text {frac }}=I-S_{\text {frac }}$ and are calculated for the open loop function with the fractional controllers $L_{(f-p i d)}$.

The sensitivity $(S)$ function address robust performance of the closedloop system. As expected, the non-integer-order systems with the highest $\mathrm{PM} \phi_{m}=75 \mathrm{deg}$ and $85 \mathrm{deg}$ has sensitivity plots with flatter peaks in the frequency range of interest compared to the integer-order PID system. Flatter peaks of $S$ with the non-integer-order controllers indicate better robust performance [22] to input disturbances compared to the integer-order one. The fractional order controllers maintain the good noise attenuation profile as well (seen via the complementary sensitivity plot).

\section{Conclusion}

A rigorous study on discrete PLC-based FOPID control design, relevant approximation aspects prior to implementation, for a process characterised by input delay was presented. In particular, an experimental testbed of a water tank volume system was used for control validation and to support the real-time design implementation. Stability of the proposed controllers was illustrated. The fractional-order discrete water volume control system designs ensure sufficient control performance despite the set-point change and system delay. The benefits of using fractional-order controllers, obtained for different approximation methods and approximation order were shown both via numerical simulation (including robustness analysis) and 
PLC-BASED DISCRETE FRACTIONAL-ORDER CONTROL 21

experimental results. The paper should be of considerable interest to process control engineers who have to provide practical solutions but may be put off by the non-conventional nature of fractional control techniques (in particular its practical PLC implementation approach). The authors are currently investigating MIMO water tank control operation.

\section{Acknowledgements}

The work is supported via University gran no.S/WM/1/2016 of Department of Automatic Control and Robotics, Bialystok University of Technology and supported by Polish Ministry of Science and Higher Education.

\section{References}

[1] Das. S., Functional fractional calculus for system identification and controls, Springer, Berlin, 2008.

[2] Girejko E., Mozyrska D., Wyrwas M., Comparison of h-difference fractional operators, Advances in the theory and applications of non-integer order systems, W. Mitkowski et al. (Eds.), 2013, pp 191-197.

[3] Kaczorek T., Selected problems of fractional systems theory, Springer, 2011.

[4] Kilbas A.A., Srivastava H.M., Trujillo J.J., Theory and applications of fractional differential equations, Elsevier, Amsterdam, 2006.

[5] I. Petráš, Tuning and implementation methods for fractional-order controllers. Fract. Calc. Appl. Anal., 15, No 2 (2012), 282-303.

[6] M. S. Tavazoei, Time response analysis of fractional-order control systems: A survey on recent results. Fract. Calc. Appl. Anal., 17, No 2 (2014), 440-461.

[7] Matasu R., Application of fractional order calculus to control theory, International Journal of Mathematical Models and Methods in Applied Sciences, 2012, 5(7), pp 1162-1169.

[8] Bastos N.R.O., Ferreira R.A.C., Torres D.F.M., Necessary optimality conditions for fractional difference problems of the calculus of variations, Discrete \& Continuous Dynam. Sys., 2011, 29/2, pp 417-437.

[9] Brzezinski D.W., Ostalczyk P., About accuracy increase of fractional order derivative and integral computations by applying the GrunwaldLetnikov formula, Communications in Nonlinear Science and Numerical Simulation, 2016, 40, pp 151-162.

[10] Nartowicz T., Pawluszewicz E., Mystkowski A., Robust fractional order controller for a linear active magnetic bearing system, 22th International Conference on Methods and Models in Automation and Robotics, Miedzyzdroje, Poland, 22nd 28-31 August, 2017. 
[11] Bandyopadhyay B., Kamal S., Stabilization and control of fractional order systems: a sliding mode approach, Lecture Notes in Electrical Engineering, Springer International Publishing, 2015.

[12] Ranganayakulu R., Uday Bhaskar Babu G., Seshagiri Rao A., Dipesh Shikchand Patle, A comparative study of fractional order $\operatorname{PI} \lambda / \operatorname{PI} \lambda \mathrm{D} \mu$ tuning rules for stable first order plus time delay processes, In ResourceEfficient Technologies, 2016, 2/1, 136-152.

[13] Agrawal O.P., Generalized multiparameters fractional variational calculus, International Journal of Differential Equations, 2012.

[14] Petras I., Fractional-Order Nonlinear Systems: Modeling, Analysis and Simulation, Springer, Series: Nonlinear Physical Science, 2011.

[15] Podlubny I., Fractional differential equations, Academic Press, San Diego, 1999.

[16] Oldham K.B,. Spaniel J., The fractional calculus, Academic Press, New York and London, 1974.

[17] Ostalczyk P., Epitome of fractional calculus, Press Lodz Lodz University of Technology, 2008.

[18] Oustaloup A., Diversity and non-integer differentiation for system dynamics, Wiley 2014.

[19] Lanusse P., Sabatier J., PLC implementation of a Crone controller, Fract. Calc. Appl. Anal., 11(4), November 2011.

[20] Machado T. J., Mainardi F., and Kiryakova V., Fractional calculus: Quo Vadimus?(Where are we going?). Fract. Calc. Appl. Anal., 18, No 2 (2015), 201-218; DOI:10.1515/fca-2015-0031.

[21] Äström, K., J., Limitations on control system performance, European Journal of Control, 2000, 6(1), pp 2-20.

[22] S. Skogestad, I. Postlethwaite, Multivariable feedback control: analysis and design. New York: Wiley, 2, (2007).

${ }^{1}$, Bialystok University of Technology

Department of Automatic Control and Robotics

Bialystok, Poland

e-mail: a.mystkowski@pb.edu.pl

2, School of Engineering

College of Science

University of Lincoln

Lincoln, LN6 7TS, UK

Received: December 2017 